\title{
Sonographic Findings of Pectoralis Major Tears With Surgical, Clinical, and Magnetic Resonance Imaging Correlation in 6 Patients
}

\author{
Jennifer S. Weaver, MD, Jon A. Jacobson, MD, \\ David A. Jamadar, MBBS, Sean E. Theisen, MD, \\ Farhad Ebrahim, MD, Monica Kalume-Brigido, MD
}

\begin{abstract}
Objective. The purpose of this research was to describe the sonographic findings of pectoralis major injuries with clinical, surgical, and magnetic resonance imaging (MRI) correlation. Methods. Images from sonographic examinations of the pectoralis major muscle of 6 patients were retrospectively evaluated and characterized. The sonographic findings were compared with clinical, surgical, and MRI findings. Results. The 6 patients were male (mean age, 30 years) with injuries sustained during weight lifting, football, and shotgun firing. Two of the 6 patients had MRI correlation; 1 had surgical correlation; 2 had both surgical and MRI correlation; and 1 had clinical follow-up. The sternal head was injured in 5 patients; 4 involved the musculotendinous junction, and 1 involved the distal tendon. The sonographic findings of muscle fiber retraction and surrounding hemorrhage allowed identification of the affected muscle. Direct impact injury causing hematoma involved the clavicular head in 1 patient. In total, 5 cases were partial-thickness pectoralis major tears, whereas complete distal tendon disruption was found in 1. Conclusions. Sonographic imaging longitudinal to the pectoralis muscle fibers showed fiber disruption, retraction, and possible hypoechoic or anechoic hematoma, most commonly involving the musculotendinous junction of the sternal head. Distal tendon assessment is important to evaluate for a full-thickness pectoralis major tear. Key words: magnetic resonance imaging; muscles, injuries; muscles, magnetic resonance imaging; muscles, sonography; sonography.
\end{abstract}

Abbreviations

MRI, magnetic resonance imaging; PD, proton density; $T E$, echo time; TR, repetition time

Received August 9, 2004, from the Department of Radiology, University of Michigan Medical Center, Ann Arbor, Michigan USA (J.S.W., J.A.J., D.A.J., F.E., M.K.-B.); and Toledo Radiological Associates, Toledo, Ohio USA (S.E.T.). Revision requested August 25, 2004. Revised manuscript accepted for publication September 7, 2004.

Address correspondence and reprint requests to Jon A. Jacobson, MD, Department of Radiology, University of Michigan Medical Center, 1500 E Medical Center Dr, TC-2910G, Ann Arbor, MI 481090326 USA.

E-mail: jjacobsn@umich.edu
7 raumatic injuries of the pectoralis major muscle in otherwise healthy individuals are increasing in frequency, which coincides with the increasing number of individuals involved in recreational sporting and athletic activities; previously, these injuries were described chiefly in debilitated elderly persons. ${ }^{1-3}$ Clinically, it is often difficult to evaluate the extent and location of these injuries, which is partially attributable to the complex anatomic characteristics (Figure 1). It is essential to differentiate between full- and partial-thickness tears because this is the crucial deciding factor between a surgical or nonsurgical repair. ${ }^{4,5}$ In addition, misdiagnosis of a pectoralis major tear can result in deformity and disability. 
Although the magnetic resonance imaging (MRI) features of pectoralis injuries have been extensively described, ${ }^{4-6}$ to our knowledge, the sonographic findings have not been fully investigated. ${ }^{7}$ In our clinical practice, sonography has been requested to evaluate the integrity of the pectoralis muscle and to evaluate for injury. The purpose of this study was to describe the sonographic findings of pectoralis major injuries with clinical, surgical, and MRI correlation.

\section{Materials and Methods}

This retrospective study was granted exemption by our Institutional Review Board. Retrospective search of radiology records identified 6 patients during the period from March 2001 through July 2002 who underwent sonographic examination of the pectoralis major muscle. Each examination was performed by 1 of 2 musculoskeletal radiologists trained and experienced in musculoskeletal sonography (11 and 8 years of experience). Sonography was performed with a $7-$ to $15-\mathrm{MHz}$ linear transducer (HDI 5000; Philips Medical Systems, Bothell, WA). Sonographic evaluation of the pectoralis muscle was completed longitudinal and transverse to the muscle and tendon fibers from the origin to insertion. The distal pectoralis tendon was identified and evaluated in the transverse plane at the level of the inferior bicipital groove of the humerus where the pectoralis tendon courses over the long head of the biceps

Figure 1. Diagram of the pectoralis major muscle shows the clavicular head (C) inserting inferior to the sternal head, which comprises manubrial (Sm) and abdominal (Sa) laminae.

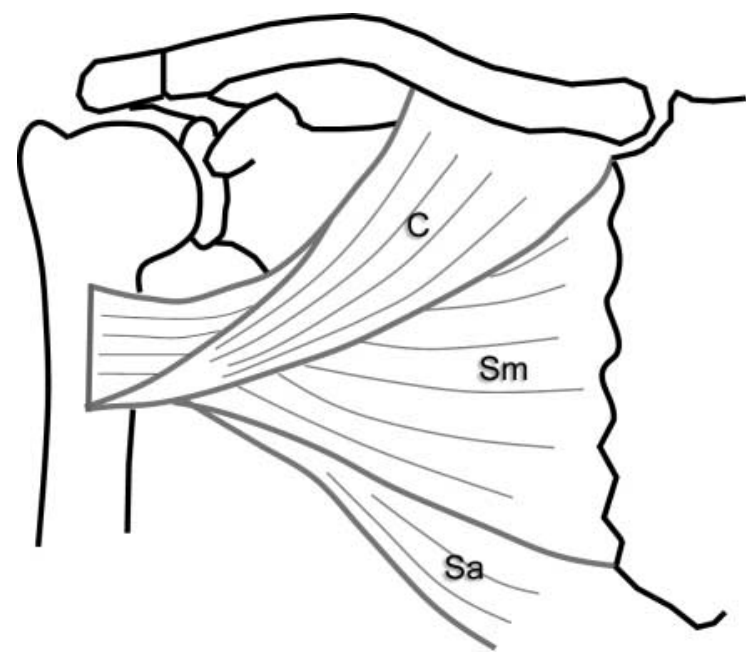

brachii tendon (Figure 1). Comparison with the contralateral asymptomatic side was often used.

Patient records were reviewed by 1 author, where it was found that 4 of the 6 patients also underwent MRI examination of the pectoralis major muscle. The studies were performed on a 1.5-T system (Signa; GE Medical Systems, Milwaukee, WI) including T1-weighted images (echo time [TE], 466-900 milliseconds; repetition time [TR], 14 milliseconds), proton density (PD)weighted fast spin echo images (TR, 2500-3000 milliseconds; TE, 14-30 milliseconds) with fat saturation, and inversion recovery sagittal, coronal, and axial images (TE, 3000-3500 milliseconds; TR, 14-30 milliseconds; inversion time, 165 milliseconds; matrix, 192-256 $\times$ 256-512; field of view, 20-48 cm). Magnetic resonance imaging examinations were either ordered by the referring physician as part of the patient's diagnostic workup or suggested by the radiologist performing the sonographic examination. Magnetic resonance imaging examinations were performed before the sonographic examination in 1 patient (interval, 20 days) and after sonographic examination in 3 (interval range, 6-110 days; average, 50 days).

The sonographic and MRI images were then retrospectively reviewed by 2 musculoskeletal radiologists trained and experienced in musculoskeletal sonography (11 and 8 years), 1 of whom was the individual who performed the sonographic examinations. Images were analyzed for site (enthesis, musculotendinous junction, or intramuscular) and extent of muscle or tendon injury (partial- or full-thickness tear). Normal tendon was characterized as hyperechoic and fibrillar on sonography and as having a uniformly low signal on MRI. Normal muscle was characterized as predominately hypoechoic with interspersed hyperechoic connective tissue on sonography and as having an intermediate signal on MRI. Abnormal tendon or muscle was characterized by hypoechoic or anechoic areas on sonography and a fluid signal on MRI. Fullthickness tears were characterized by complete tendon or muscle fiber disruption with retraction. The presence or absence of hematoma was noted, characterized by anechoic or hypoechoic areas or heterogeneous abnormal mixed echogenicity with a mass effect on sonography and a fluid signal on MRI. The imaging findings were compared with clinical and surgical findings (where available). 


\section{Results}

\section{Patients}

The 6 patients were male with a mean age of 30 years (range, 15-44 years). Three of the 6 patients had injuries sustained during weightlifting; 1 had an injury sustained during a football tackle; 1 had a direct impact injury from the repetitive firing of a 12-gauge shotgun; and the sixth had an unknown mechanism of injury. The time between injury and sonography ranged from 7 days to 5 months. Two of the 6 patients had MRI correlation; 1 had surgical correlation; 2 had both surgical and MRI correlation; and 1 had clinical follow-up.

The 1 patient with clinical follow-up had hematoma and partial-thickness disruption within the clavicular head of the pectoralis major muscle (Figure 2). Of the 5 patients with MRI correlation, surgical correlation, or both, all had involvement of the pectoralis major sternal head. In 4 of these, the abnormality was at the musculotendinous junction; 3 were isolated full-thickness tears of the sternal head with sparing of the clavicular head (Figure 3), and 1 was a full-thickness tear of the sternal head with an additional partial-thickness tear of the clavicular head (Figure 4). In the fifth patient with MRI correlation, surgical correlation, or both, there was a fullthickness tear of the sternal and clavicular heads at the humeral attachment (Figure 5), representing the only full-thickness pectoralis major tear in which both heads were completely disrupted.

\section{Image Evaluation}

In the 4 patients with musculotendinous junction tears of the sternal head, the sonographic findings included fiber disruption at this site and muscle retraction (Figure 3). Surrounding anechoic or hypoechoic hemorrhage was also present, outlining the retracted torn muscle and tendon stump. In 1 of these patients, there was incomplete extension of the tear into the clavicular head (Figure 4). In each case, at least 1 portion of the distal pectoralis major tendon was identified as a continuous hyperechoic structure superficial to the biceps brachii long head tendon extending from the musculotendinous junction; therefore, complete full-thickness pectoralis tendon tear was excluded.

In the 1 patient with a full-thickness pectoralis tendon tear at the humeral shaft, there was muscle retraction more proximally (Figure 5). In this

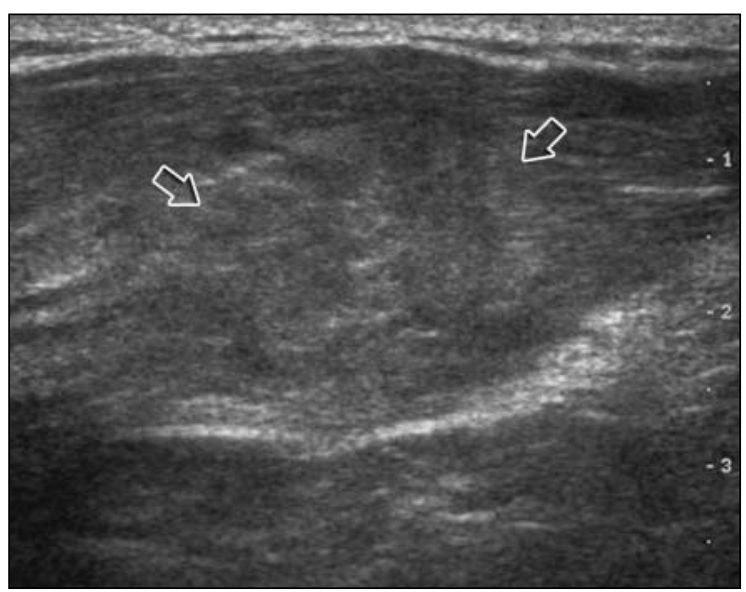

Figure 2. Partial-thickness tear and hematoma of the clavicular head with an intact sternal head (partial-thickness pectoralis major tear). Sonography longitudinal to the clavicular head shows a mass effect from the heterogeneous and nearly isoechoic hematoma (arrows)

case, there was no soft tissue hemorrhage outlining the retracted muscle (the interval between injury and imaging was 12 weeks). In addition, the distal pectoralis tendon was not visible superficial to the long head of the biceps brachii tendon at the humeral insertion.

In the 1 patient with intramuscular injury to the clavicular head with hematoma, a heterogeneous masslike area nearly isoechoic to muscle was seen disrupting the normal muscle echo texture (Figure 2). The sternal head was intact, and distal tendon attachment to the humerus was normal.

\section{Follow-up}

The 3 patients with surgical correlation of the partial-thickness pectoralis major tears went on to have direct surgical repair. In the 1 patient with distal tendon disruption at the humeral shaft, only partial repair was achieved because the degree of tendon retraction in the setting of the full-thickness sternal and clavicular heads did not allow complete apposition at the site of the tear. The 1 patient with clinical correlation of the clavicular head injury was asymptomatic after 2 years of clinical follow-up.

\section{Discussion}

Our results show that sonography can depict injuries to the pectoralis major muscle and tendon. Complete musculotendinous disruption and retraction characterized a full-thickness tear of a muscle head; however, tendon fiber continu- 
ity from the remaining muscle head excluded a full-thickness tear of the pectoralis major muscle.

The pectoralis major muscle is important in function; this muscle adducts, flexes, and internally rotates the arm. It has a broad origin from the clavicle, sternum, and costal cartilages; the muscle fibers converge into 3 laminae that rotate $180^{\circ}$ to coalesce into a tendon that inserts into

Figure 3. Full-thickness tear of the sternal head with an intact clavicular head (partial-thickness pectoralis major tear). Sonography longitudinal to the pectoralis major sternal head at the musculotendinous junction (A) and distal (B) with corresponding axial PDweighted fast spin echo MRI with fat saturation (C) show fiber disruption and retraction (filled arrow) and fluid (open arrows) at the expected site of the distal tendon. Sonography longitudinal to the clavicular head (D) shows intact fibers (filled curved arrows). Evaluation of the asymptomatic contralateral side longitudinal to the distal pectoralis tendon $(\mathbf{E})$ shows an intact distal tendon (open curved arrows). Note the long head of biceps brachii tendon (arrowhead), coracobrachialis muscle (CB), and humerus $(\mathrm{H})$.

\section{B}

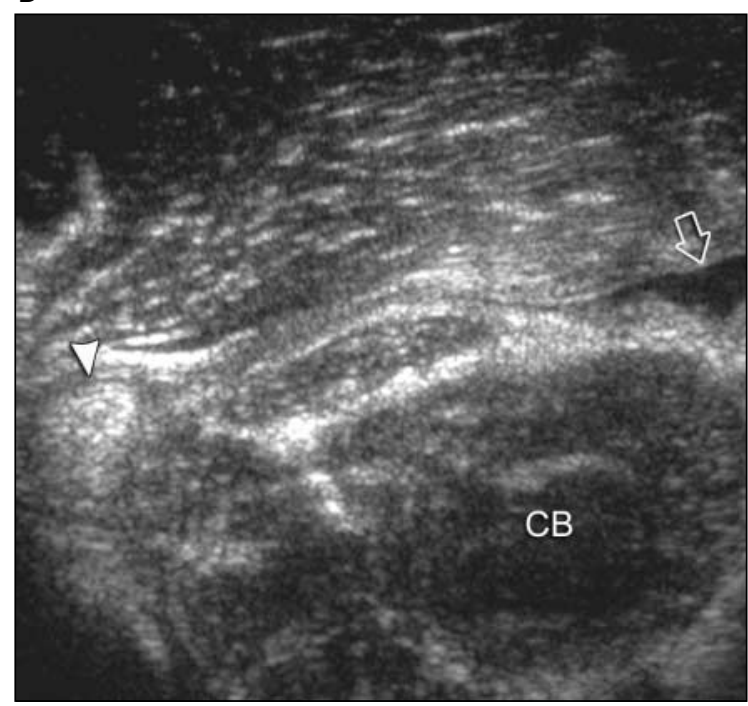

D

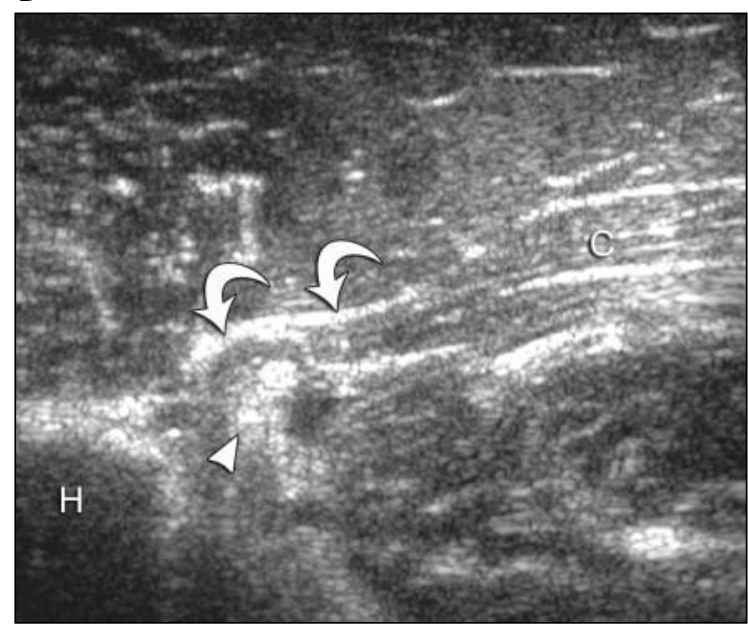

the bicipital groove of the humerus. ${ }^{6}$ The clavicular head of the pectoralis major muscle consists of fibers from the clavicular lamina, originating from

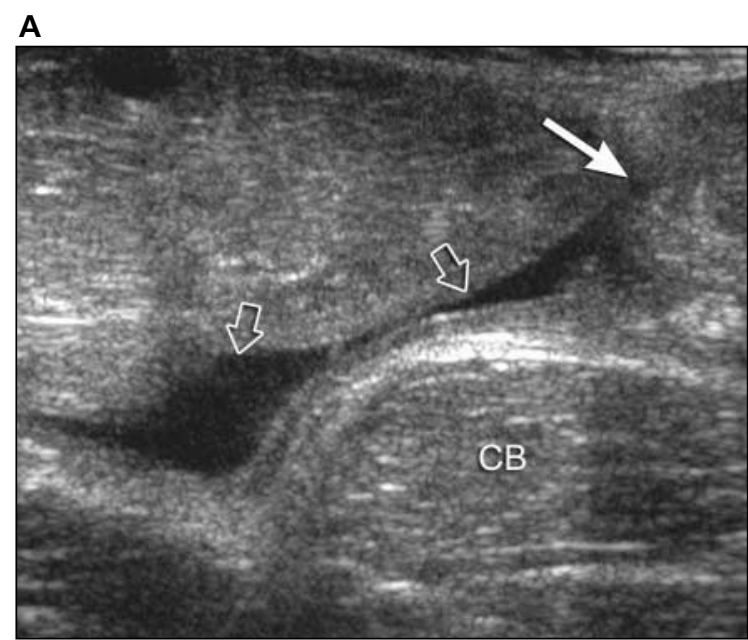

C

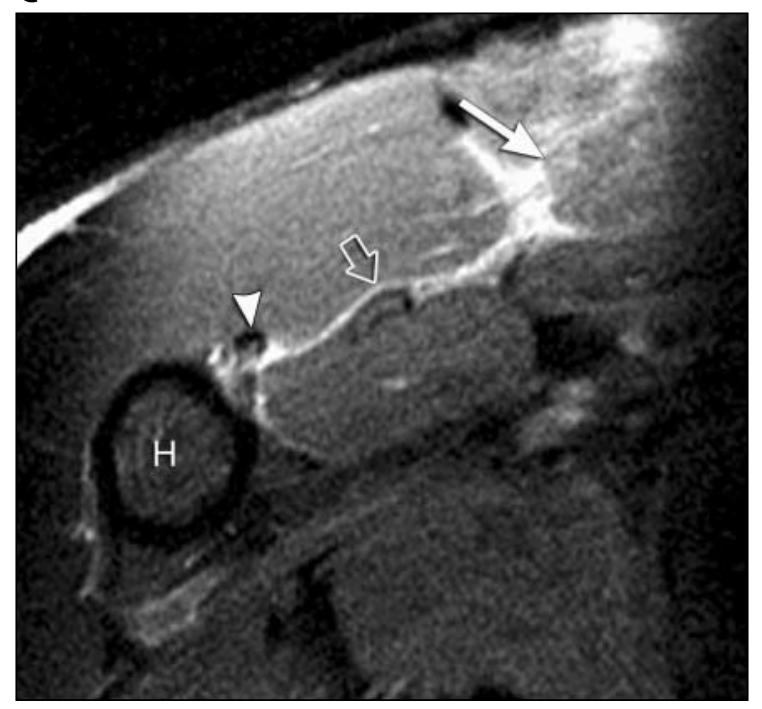

E

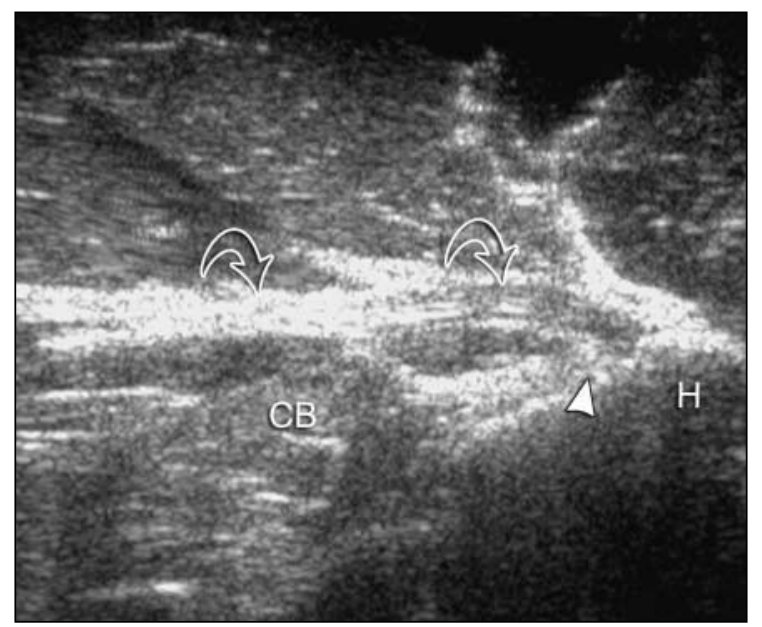


the medial two thirds of the clavicle; these fibers insert distally and anteriorly. The sternal head of the pectoralis major muscle consists of fibers from the abdominal and manubrial laminae; these fibers combine and course deep to the clavicular head to insert on the humerus superior to the clavicular head. ${ }^{4}$ In total, the cephalocaudad dimension of the distal tendon insertion on the humerus is approximately 4 to $6 \mathrm{~cm} .{ }^{6}$ The most superior extent of the tendon insertion is at or 1 to $1.5 \mathrm{~cm}$ inferior to the quadrilateral space and 5 to $10 \mathrm{~mm}$ superior to the lateral head of the triceps tendon, whereas the inferior extent of the tendon is typically superior to the deltoid attachment. ${ }^{6}$
Tears of the pectoralis major muscle have been described in debilitated elderly persons ${ }^{1-3}$; however, traumatic injuries of the pectoralis major muscle are increasing in frequency in otherwise healthy individuals with the recent increase in participation in recreational sporting activities. This injury has been reported in weight lifters, football players, wrestlers, and water skiers, although one of the earliest descriptions of this injury occurred in a butcher lifting a large piece of beef. ${ }^{5}$ The mechanism of injury involves excessive stress with the muscle under full tension or a direct blow with the arm in abduction and extension. Direct impact injuries, such as repetitive shotgun firing, can also cause injury to

Figure 4. Full-thickness tear of the sternal head with a partial-thickness tear of the clavicular head (partial-thickness pectoralis major tear). Sonography (A) and PD-weighted axial MRI (B) of the sternal head musculotendinous junction show fiber disruption and retraction (filled arrows). Sonography longitudinal to the clavicular head (C) and coronal PD-weighted MRI (D) show partial-thickness disruption of the clavicular head (open arrows). Note the sternal head (S), clavicular head $(\mathrm{C})$, humerus $(\mathrm{H})$, and intact distal clavicular head (filled curved arrow).

A

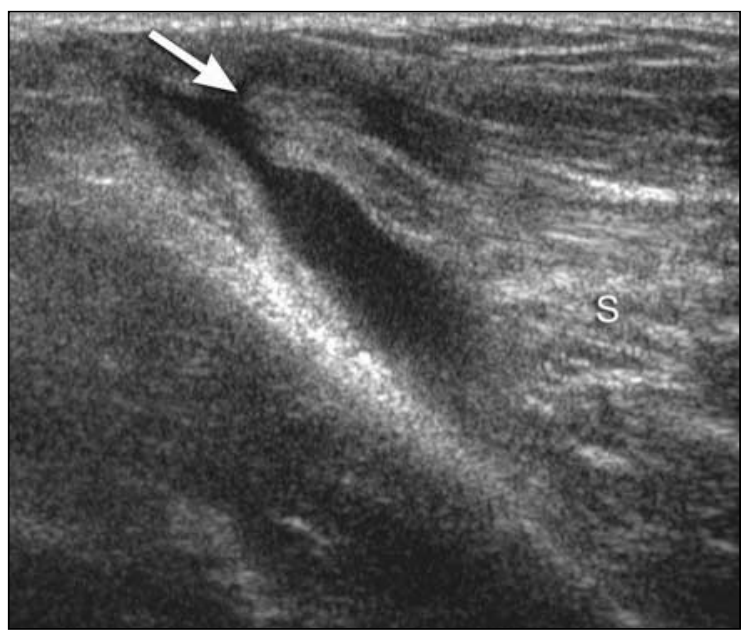

C

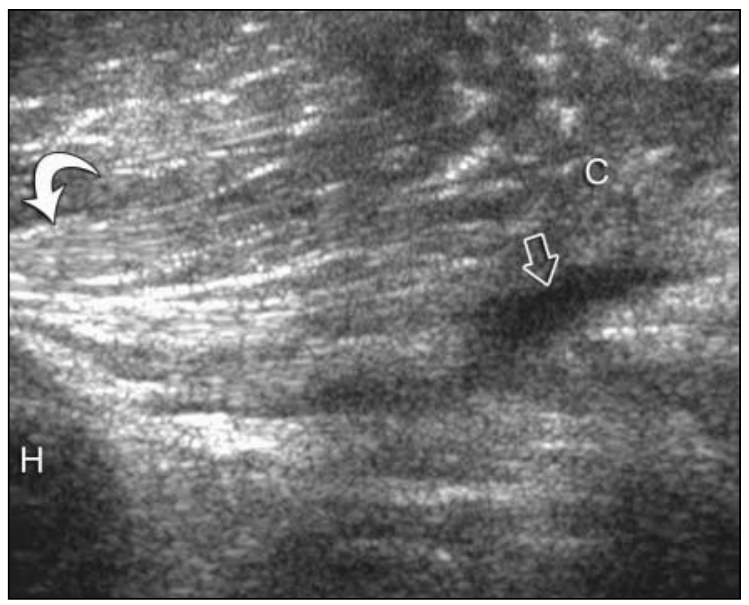

B

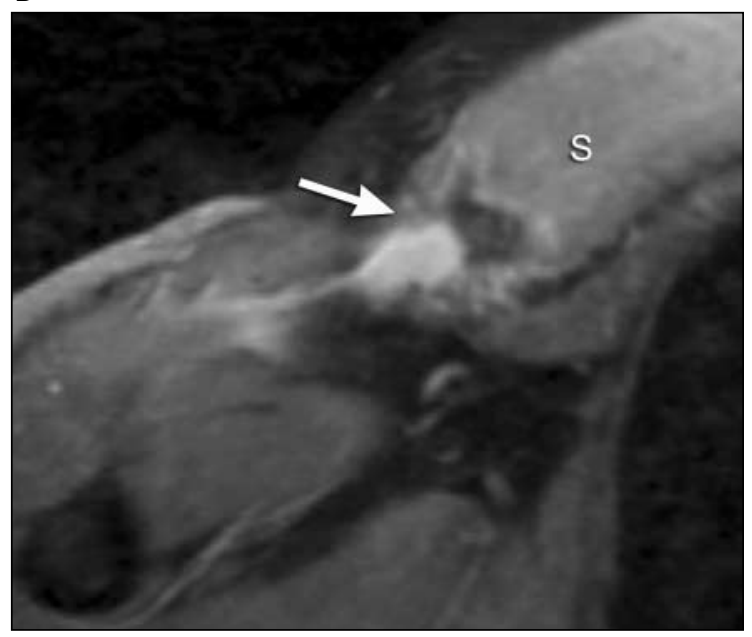

D

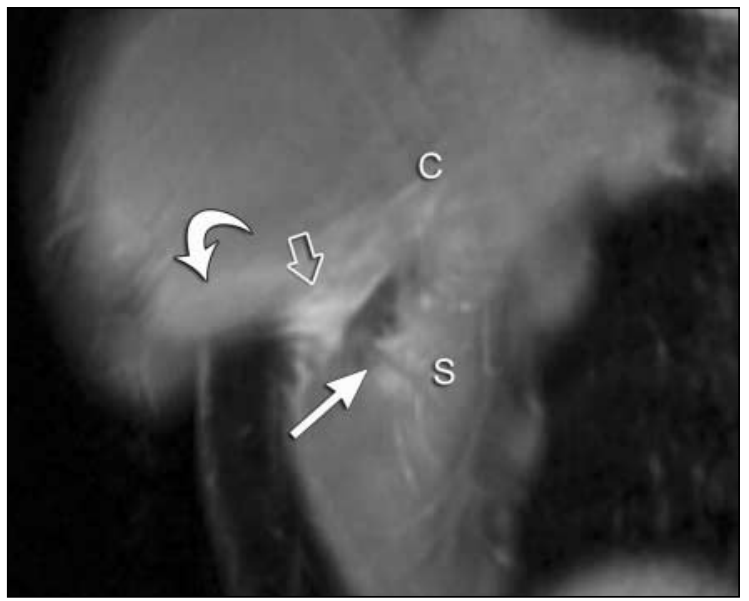


Figure 5. Full-thickness tear of the sternal and clavicular heads (full-thickness pectoralis major tear). Sonography (A) and longitudinal relaxation time-weighted (B) and inversion recovery $(\mathbf{C})$ axial MRI show distal pectoralis major (Maj) fiber disruption and retraction (filled arrow) and no visualization of distal the tendon (open arrows). Note the pectoralis minor muscle (Min) and rib (R).

\section{A}

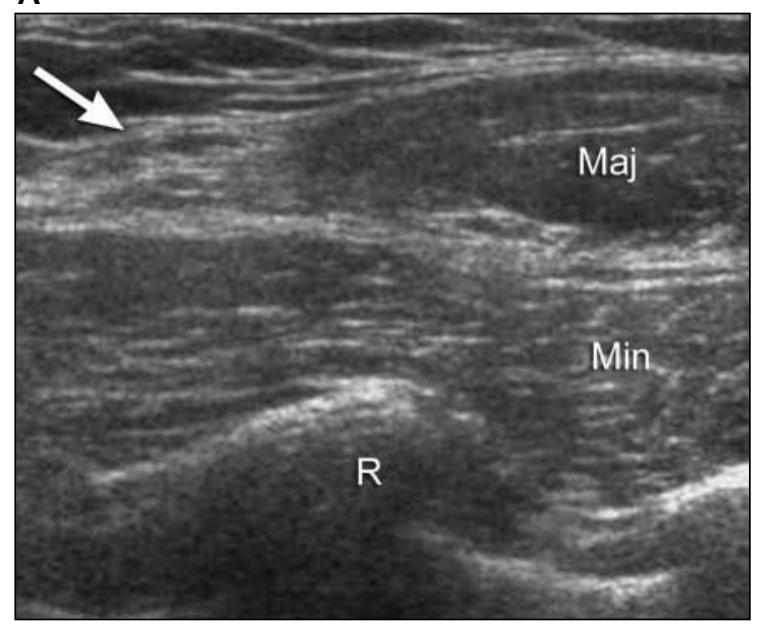

\section{B}

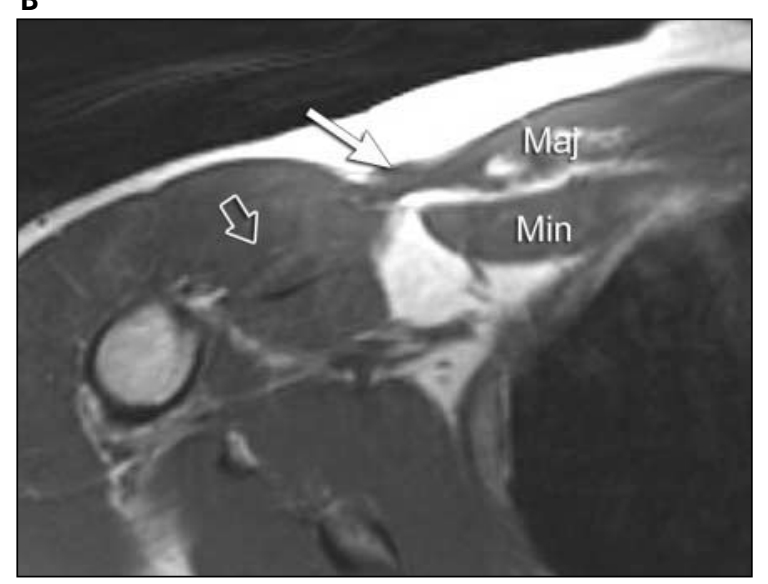

C

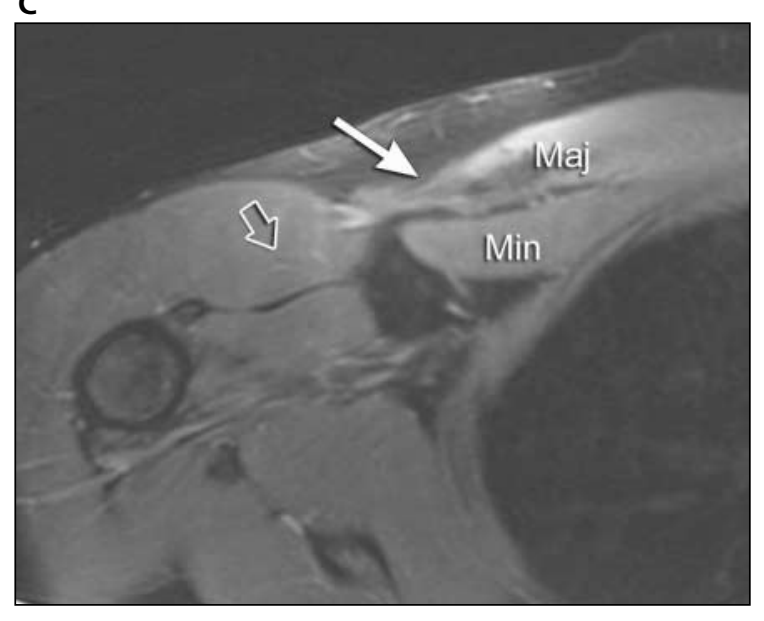

the pectoralis major muscle. Patients with injuries of the pectoralis major muscle often will have pain, ecchymosis, edema, and decreased range of motion. It is difficult to evaluate the extent and location of these injuries clinically.

Pectoralis major muscle injuries are classified by degree as either partial- or full-thickness tears. Injuries can be located at the enthesis, the myotendinous junction, or intramuscularly. ${ }^{5}$ Partial-thickness tears are the most common, usually involving the musculotendinous junction. The sternal head is prone to tear first as its abdominal laminae inserted highest on the humerus are disproportionately stretched with terminal humeral extension; the tear may then extend into the clavicular head. ${ }^{8}$ Sparing of the clavicular head limits the extent of sternal head retraction. ${ }^{8}$ Those injuries involving the muscle belly are usually from direct impact. Full-thickness tears usually involve the distal tendon or the attachment of the tendon to bone. ${ }^{4}$

Although MRI has historically been used to evaluate injuries of the pectoralis major muscle, ${ }^{4-6}$ sonography can be used to characterize these injuries as well. Although 1 prior report described hypoechoic hematoma in the setting of pectoralis major tears, ${ }^{7}$ the role of sonography in differentiating full-thickness from partialthickness tears and the ability of sonography to characterize injury to each individual muscle head has not been fully investigated. The results of our study show that the sonographic findings of injuries to the pectoralis major muscle include disruption of the musculotendinous junction, muscle fiber retraction, and possible surrounding anechoic or hypoechoic hemorrhage. In 1 patient, no fluid or hematoma was seen at the retracted tendon tear. The distal tendon attachment at the humerus extending from the musculotendinous junction was at least partially identified with the partial-thickness tears and not visualized with the full-thickness tear.

With regard to sonographic technique in evaluation of the pectoralis major muscle, imaging in the longitudinal plane relative to each muscle head will show fiber disruption and retraction if present, particularly at the musculotendinous junction. It is important to accurately identify and completely examine the distal pectoralis tendon. The distal tendon is identified in the transverse plane coursing superficially to the long head of the biceps brachii tendon inferior to the level of the subscapularis tendon. Although it 
is difficult to separate the individual tendon heads at this level, the sternal head inserts superior to the clavicular head. Evaluation of the entire cephalocaudad extent of the distal tendon is imperative; although complete tendon disruption may be seen in a single area, intact fibers more superiorly or inferiorly exclude a full-thickness pectoralis tear.

Treatment is typically nonsurgical for partialthickness tears and occasionally for full-thickness tears in nonathletes. ${ }^{9,10}$ Surgical repair is used for full-thickness tears, tears involving the distal tendon, and injuries in athletes. Several perils of delayed diagnosis exist, including adhesions, muscle retraction, muscular scar and fibrosis, atrophy, and overall poor surgical outcome.

We acknowledge several limitations to this study, which include the small sample size and lack of surgical or MRI correlation in 1 patient; therefore, the overall accuracy of sonography in the diagnosis of pectoralis major tears was not assessed. In addition, a potential selection bias exists because those patients who are most symptomatic are more likely to seek medical attention. Last, sonographic findings of pectoralis tears were characterized from static images, whereas sonographic diagnoses often rely on real-time scanning.

In summary, most injuries of the pectoralis major muscle involved the musculotendinous junction of the sternal head, characterized by muscle fiber disruption, retraction, and possible anechoic or hypoechoic hemorrhage. Integrity of the distal tendon and musculotendinous junction of each individual muscle head allowed classification of the extent of the injury as either a partial- or full-thickness pectoralis major tear.

\section{References}

1. Beloosesky $Y$, Hendel $D$, Weiss A, Rosenberg PH, Grinblat J. Rupture of the pectoralis major muscle in nursing home residents. Am J Med 2001; 111:233235.

2. Beloosesky $Y$, Grinblat J, Hendel D, Sommer R. Pectoralis major rupture in a 97-year-old woman. J Am Geriatr Soc 2002; 50:1465-1467.

3. Povoski SP, Spigos DG. Partial tear of pectoralis major muscle masquerading as a breast mass in an 87-yearold woman. Acta Radiol 2002; 43:615-616.
4. Connell DA, Potter HG, Sherman MF, Wickiewicz TL. Injuries of the pectoralis major muscle: evaluation with MR imaging. Radiology 1999; 210:785-791.

5. Carrino JA, Chandnanni VP, Mitchell DB, ChoiChinn DB, DeBerardino TM, Miller MD. Pectoralis major muscle tendon tears: diagnosis and grading using magnetic resonance imaging. Skeletal Radiol 2000; 29:305-313.

6. Lee J, Brookenthal KR, Ramsey ML, Kneeland JB, Herzog R. MR imaging assessment of the pectoralis major myotendinous unit: an MR imaging-anatomic correlative study with surgical correlation. AJR Am J Roentgenol 2000; 174:1371-1375.

7. Beloosesky Y, Grinblat J, Katz M, Hendel D, Sommer R. Pectoralis major rupture in the elderly: clinical and sonographic findings. Clin Imaging 2003; 27:261264.

8. Anbari A, Kelly JD, Moyer RA. Delayed repair of a ruptured pectoralis major muscle: a case report. Am J Sports Med 2000; 28:254-256.

9. Quinlan JF, Molloy M, Hurson BJ. Pectoralis major tendon ruptures: when to operate. Br J Sports Med 2002; 36:226-228.

10. Dodds SD, Wolfe SW. Injuries to the pectoralis major. Sports Med 2002; 32:945-952. 